\title{
Nonlinear Data Mining of Microarray Data Using Michaelis Menten
} Functions

\author{
Dov J Stekel*, Tim W Overton, Jon L Hobman, Charles W Penn, Jeff A Cole \\ and Chrystala Constantinidou
}

Address: School of Biosciences, University of Birmingham, UK.

Email: Dov J Stekel* - d.j.stekel@bham.ac.uk

* Corresponding author

from BioSysBio: Bioinformatics and Systems Biology Conference

Edinburgh, UK, 14-15 July 2005

Published: 2 I September 2005

BMC Bioinformatics 2005, 6(Suppl 3):SI3

One of the great challenges of Systems Biology is the reconstruction of biological processes from "omics" data, such as that generated by DNA microarrays, proteomics or metabolomics. Within the field of microarray data analysis, it is common practice to explore the relationships between similar genes using techniques such as cluster analysis or principal component analysis, and increasingly common to employ reverse engineering techniques such as probabilistic graph models (Bayesian networks). In both cases, it is essential to model the relationship between gene expression profiles. Current methodologies use linear models, either parametrically (e.g. Pearson correlation) or non-parametrically (e.g. Spearman correlation).

However, it is well established that the kinetics of core biological processes - transcription, metabolism and signalling - are not linear, but follow non-linear MichaelisMenten kinetics. In this work, we use nonlinear least squares to fit Michaelis-Menten functions to gene expression profiles. The technique is sufficiently flexible to fit linear relationships when they are present, as well as nonlinear relationships representing either activation or repression. As an example, we demonstrate the technique applied to an extensive series of experiments aimed at understanding anaerobic respiration in wild-type and FNR-mutant E. coli. We demonstrate that this technique uncovers relationships that would not have been found using standard linear techniques. We show that the nonlinear relationships are found precisely in those parts of the network associated with respiration. Many of these relationships come from known processes; some of these relationships are novel. We conclude that it is important to apply mechanistic nonlinear techniques in the analysis of microarray data. 